Title

\title{
The somatotopy of observed emotions
}

${ }^{1}$ Alejandra Sel ${ }^{* 1,2,3}$, Beatriz Calvo-Merino ${ }^{1}$, Manos Tsakiris ${ }^{3}$, Bettina Forster ${ }^{1}$

${ }^{1}$ Cognitive Neuroscience Research Unit, Department of Psychology, City University London.

Northampton Square, EC1V 0HB, London, U.K

${ }^{2}$ Department of Psychology, University of Essex, Wivenhoe Park, Colchester, CO4 3SQ, UK.

${ }^{3}$ Lab of Action \& Body, Department of Psychology, Royal Holloway University London,

Egham, Surrey, TW20 0EX, London, U.K.

\section{Corresponding author:}

Alejandra Sel

Cognitive Neuroscience Research Unit

Department of Psychology

City University London. Northampton Square, EC1V 0HB, London, U.K

alex.sel@essex.ac.uk

${ }^{1}$ Present Address:

Centre for Brain Science

Department of Psychology, University of Essex

Wivenhoe Park, Colchester, CO4 3SQ

Tel: +44 (0)1206 873817 


\begin{abstract}
The ability to experience others' emotional states is a key component in social interactions. Uniquely among sensorimotor regions, the somatosensory cortex (SCx) plays an especially important role in human emotion understanding. While distinct emotions are experienced in specific parts of the body, it remains unknown whether the SCx exhibits somatotopic activations to different emotional expressions. In the current study, we investigated if the affective response triggered by observing others' emotional face expressions leads to differential activations in SCx. Participants performed a visual facial emotion discrimination task while we measured changes in SCx topographic EEG activity by tactually stimulating two body-parts representative of the upper and lower limbs, the finger and the toe respectively. The results of the study showed an emotion specific response in the finger SCx when observing angry as opposed to sad emotional expressions, after controlling for carryover effects of visual evoked activity. This dissociation to observed emotions was not present in toe somatosensory responses. Our results suggest that somatotopic activations of the SCx to discrete emotions might play a crucial role in understanding others' emotions.
\end{abstract}

Keywords: emotions, somatosensory cortex, embodiment, EEG, somatotopic responses 


\section{Introduction}

Humans possess the unique ability to experience others' emotional states, and to utilize these embodied responses to understand and predict behaviors in complex social interactions (Bastiaansen, Thioux, \& Keysers, 2009; Hess \& Fischer, 2014; Niedenthal, 2007). The experience and understanding of observed emotions are supported by a distributed brain network comprising low-level sensory areas in visual, auditory and motor cortices, as well as high-level brain areas such as the prefrontal and orbitofrontal cortices, and the temporo-parietal cortex (Atkinson \& Adolphs, 2011; Baseler, Harris, Young, \& Andrews, 2012; Engell \& Haxby, 2007). Among these areas, the motor and sensory cortices including the somatosensory cortex ( $\mathrm{SCx})$ are critical areas for action representation being highly interconnected to the limbic system. Activity in these areas is linked to performance in simple perceptual tasks such as emotion recognition or discrimination of facial and body expressions, as well as more complex social tasks such as imitation or perspective taking tasks (Carr, Iacoboni, Dubeau, Mazziotta, \& Lenzi, 2003; Nakamura et al., 1999). Importantly, this line of work highlights the role of the observer's body in others' emotion understanding.

The involvement of the body in the experience of one's emotion was first proposed by William James (1884) and his contemporaneous colleague Carl Lange (1885/1912). They postulated that physiological and behavioral responses precede subjective experience of emotions marked by "distinct bodily expressions". Since these initial observations, a substantial number of theories have been proposed to explain the role of the body in the experience of emotions. For example, it is believed that the subjective experience of emotion relies on the sensory detection of the affective peripheral feedback and its somatosensory representations. Thus, each emotion mechanism is thought to produce a distinctive internal feeling in a way that emotions, such as anger or sadness, represent universal categories of 
bodily experience (Barrett, 2006; Damasio Antonio et al., 1996). More recently, a series of behavioral studies have revisited the idea that individual emotions are associated to distinctive patterns of bodily feelings. These studies suggest that our conscious emotional experience rely on topographically distinct bodily sensations that are unique for each emotion (Nummenmaa, Glerean, Hari, \& Hietanen, 2014). These bodily sensations associated with each emotion relate to general physiological changes such as augmented activity in the upper bodily areas related to higher breathing and heart rate (Levenson, 2003), and muscular tension. Thus, for example sensations in the upper bodily areas, including the trunk, the arms, and especially the hands, were mostly linked to action-oriented emotions such as anger (Hammer \& Marsh, 2015); whereas low-arousing emotions such as sadness were related to decreased sensations in the lower bodily areas especially the feet.

This dissociation between emotion and bodily part in anger and sadness were not only found to the subjective experience of one's emotion to emotional items including words, stories and movies, but also when judging the felt bodily sensations experienced by persons depicting facial expressions (i.e. the experience of others' emotion). Therefore, these topographically distinct bodily sensations of emotion could contribute to the generation of embodied responses to observed emotions. These bodily maps of emotions have been proven to be true across cultures (Nummenmaa, Hari, Hietanen, \& Glerean, 2018), evolving during the life-span (Volynets, Glerean, Hietanen, Hari, \& Nummenmaa, In press), and they are influenced by mental diseases such as schizophrenia (Torregrossa et al., 2018). Overall, these studies support the idea that the human body is not only necessary for expressing emotions, but also important to feel one's own and others' emotional states.

In line with the subjective bodily maps of emotions, neuroimaging findings have observed specific patterns of cluster brain activity linked to groups of emotions. In particular, these studies show that one's subjective experience of emotions is instantiated in activation 
patterns in brain areas engaging the prefrontal cortex, frontal pole, cingulate cortex as well as the precentral and postcentral gyrus (Saarimäki et al., 2018; Saarimäki et al., 2015).

Interestingly, these findings show a highly consistent activation of the sensorimotor cortices, particularly to emotions related with action preparation and motor tendencies (Saarimäki et al., 2018), indicating a greater action preparation in 'fight-or-flight' type emotions. However, the pattern classification approach used in these studies failed to confirm or refute whether the actual neural organization of each emotion is represented in a somatotopic manner. Instead, they explain that the brain encoding of one's response to others' facial emotions is supported by a general activation of somatic states not linked to particular bodily areas (Nummenmaa, Hirvonen, Parkkola, \& Hietanen, 2008), leaving an open question as to whether there is a direct link between consciously experienced regional bodily sensations and discrete somatotopic activations in sensory cortices.

Uniquely among sensorimotor cortices, the right SCx has a crucial and independent role in understanding observed emotions (Atkinson \& Adolphs, 2011; Pitcher, Garrido, Walsh, \& Duchaine, 2008; Sel, Forster, \& Calvo-Merino, 2014). Thus, damage to the somatosensory cortices, as well as virtual lesions after brain stimulation, lead to an impairment in facial emotion recognition (Pitcher et al., 2008; Pourtois et al., 2004). In addition, general activations in somatosensory areas have been linked to conscious experience of others' emotions during emotion understanding tasks (i.e. representing others' feeling as opposed to experiencing our own feelings) (Saarimäki et al., 2015). In sum, these findings suggest that emotional simulation involving SCX is not purely conceptual but involves the representation of the actual body (Keysers, Kaas, \& Gazzola, 2010). The remaining question is whether the SCx exhibits distinctive patterns of somatotopic activations to different emotional expressions, resembling bodily maps of activations linked to subjective feelings. 
Here we investigate if the SCx representation goes beyond observed expressions and it contributes to the representation of others' emotional experiences. Specifically, we explore if the felt bodily sensations associated with the representation of others' facial emotions map onto specific somatotopic body-parts in SCx, in a similar way to the representation of one's own emotional experience. To examine whether SCx shows discrete somatotopic activations when observing others' facial expressions, we directly measured somatosensory-evoked responses by probing activity in separate SCx locations during a visual emotion discrimination task. We tactually probed somatosensory processing in two body-parts (i.e. left index finger and first toe -left foot, as representative areas of upper and lower bodyparts), while participants viewed angry, sad or neutral faces. This allowed to directly measure the involvement of two categorical body-parts, finger/toe, in the observation of two discrete emotions, anger/sadness. Touch to the finger and the toe leads to somatosensory evoked potentials with a morphology that allows observing changes in response amplitude, in comparison to other areas such as the upper arm or the knee. Importantly, by tapping on the left finger and left toe with tactile stimulation we could precisely probe the finger and toe somatotopic representations in the right somatosensory cortex. Previous evidence has shown that particularly the right (vs left) somatosensory cortex has an important role in emotional facial recognition (Pitcher, Garrido, Walsh, \& Duchaine, 2008; Sel, Forster, \& Calvo-Merino, 2014; Vuilleumier \& Pourtois, 2007). Therefore, the left finger and toe are likely to be good candidates for the study of discrete somatotopic activations in upper and lower bodily areas when observing others' facial expressions. To isolate the response of SCx over and above the effects induced by other processing regions, we subtracted purely visually-evoked potentials (VEPs; visual-only condition) from tactually probed somatosensory evoked potentials (SEPs; visual-tactile finger/toe conditions; Fig.1) during facial processing (Sel et al., 2014), and we 
called this "VEP-free SEPs". In addition, we tested if the changes in finger/toe somatosensory response related to the self-reported bodily sensations in upper/lower limbs.

If observing others' angry and sad emotional expression engages emotion-specific patterns of bodily activation in finger and toe, we will expect amplitude differences in tactileevoked somatosensory activity when contrasting responses to angry vs. neutral faces, and sad $v s$. neutral faces, and this emotion-specific pattern will be different in finger as opposed to toe evoked responses. More specifically, in accordance to the findings reported by Nummenmaa and colleagues $(2014 ; 2018)$ that suggest opposite activations of the upper limbs including the fingers to anger (increased activation) vs. sadness (decreased activation), we will expect divergence amplitudes in finger SCx when contrasting VEP-free SEPs to angry $v s$. sad faces which might be related to the opposite changes in perceived bodily sensations (activation $v s$. deactivation to anger and sadness, respectively). The bodily maps of emotions also show a decreased activation in the lower limbs including the toes to sadness, and a subtle activation increase to anger. Therefore, it might be expected that angry vs. sad faces could also lead to distinctive amplitude changes in toe VEP-free SEPs with greater amplitude for sadness as opposed to anger, in line with the reported bodily changes. Based on previous somatosensory research, it is likely that the amplitude changes in finger VEP-free SEPs will be observed over centro-lateral electrode sites with greater responses in the hemisphere contralateral to the side of the stimulation (i.e. right hemisphere). We might expect the amplitude changes in toe VEP-free SEPs to be most predominant over the midline electrodes, where toe SEPs are typically observed (Miller, 2012; Shen, Smyk, Meltzoff, \& Marshall, 2018; Xiang et al., 1997).

Importantly, any observed changes in VEP-free SEPs could not be explained by carry over emotion effects from visual regions. If changes in VEP-free SEPs were a byproduct of emotion effects from visual cortex, tactually evoked responses should not differ after 
removing purely visually-evoked potentials. One previous investigation has showed changes in early VEP-free SEPs to observation of fearful and happy faces - i.e. 40 to $80 \mathrm{~ms}$ after tactile onset (Sel et al., 2014). In addition, a number of studies have shown early modulations of SEPs (from 25ms after tactile onset) to observation of emotional faces (Montoya \& Sitges, 2006; Ravaja, Harjunen, Ahmed, Jacucci, \& Spapé, 2017). Therefore, we could expect modulations of VEP-free SEPs to angry and sad faces in early somatosensory responses. However, based on evidence from the visual domain, the latency of cortical responses to observed emotions rely on the emotional valence and saliency of the stimuli (Balconi \& Pozzoli, 2003). For example, while observing fearful faces is associated to amplitude changes starting $170 \mathrm{~ms}$ after the visual onset, angry and sad faces are linked to changes in the midlatencies from around 250ms after stimuli onset (Williams et al., 2004; Williams et al., 2006). Therefore, if observing others' angry and sad emotional faces involves bodily activation in finger and toe, we might expect changes in VEP-free SEPs at early but also at mid-latencies, e.g. after $80 \mathrm{~ms}$.

In addition, we expect that observing emotional expressions as opposed to neutral expressions will lead to changes in amplitude of the early and mid-latency VEPs. Thus, previous evidence has shown enhanced amplitude in the N250 component over temporooccipital sites in response to angry vs neutral faces (Schupp 2004). Similar studies have shown greatest amplitude augmentation to high-salient negative emotions such as fear or anger, in comparison to low-arousal expressions such as sadness from $230 \mathrm{~ms}$ after stimulus onset over temporo-occipital sites (Balconi \& Pozzoli, 2003). In line with this evidence, we predict enhanced amplitude at early and mid-latency VEPs to observation of emotional faces, particularly to angry faces, in comparison to neutral faces.

\section{Material and methods}


We report the procedure followed to determine the sample size, all data exclusions, all inclusion/exclusion criteria, whether inclusion/exclusion criteria were established prior to data analysis, all manipulations, and all measures in the study. None of the study procedures or analyses was pre-registered in a time-stamped, institutional registry prior to the research being conducted. However, it is worth noting that in the current study we computed the sample size and planned the analysis based on previous studies that have used the same ERP subtraction method to isolate somatosensory responses from visual processing (Arslanova, Galvez-Pol, Calvo-Merino, \& Forster, 2019; Galvez-Pol, Calvo-Merino, Capilla, \& Forster, 2018; Galvez-Pol, Calvo-Merino, \& Forster, 2020; Galvez-Pol, Forster, \& Calvo-Merino, 2018), including one study that investigated somatosensory responses to emotional faces in a very similar fashion than in the current study (Sel et al., 2014), and one study investigating modulations of somatosensory evoked responses to emotional faces (Montoya \& Sitges, 2006).

\subsection{Participants}

Participants were recruited through the online participant recruitment scheme of City University London. Inclusion and exclusion criteria were established previous to data acquisition. Eligibility required that participants were right-handed (Oldfield, 1971), aged 1845, and had no neurological history. Twenty-five healthy, right-handed participants with normal or corrected-to-normal vision took part in the experiment. Four participants were excluded from the analysis due to temporary failure of the lab settings during data collection, and three participants were excluded due to excess artifacts in the EEG signal resulting in a total of 18. All participants gave informed consent, with approval by the Research Ethics Committee, Psychology Department, City University London.

\subsection{Stimuli and procedure}


A set of 90 pictures depicting anger, sadness and neutral emotions was initially taken from the Karolinska Directed Emotional Faces set (Lundqvist, Flykt, \& Öhman, 1998). Faces were grayscaled and enclosed in a rectangular frame (140x157 inch) excluding most of the hair and non-facial contours. All facial stimuli were normalized in their visual properties such as contrast, luminance and intensity. To ensure that the facial emotional expressions effectively triggered an emotional response, eight volunteers, none of whom participated in the subsequent study, judged the strength of emotion expressed in the faces on a continuous visual analogue scale $(100=$ "extremely emotional"; $0=$ "not emotional at all"). Based on these judgments, we selected 40 emotional faces (20 angry faces $-M=70.86, S D=17.91 ; 20$ sad faces $-M=62.03, S D=21.65)$, and 20 neutral faces rated closest to the "non-emotional at all" judgment $(M=14.11, S D=15.62)$ (half male).

Tactile stimulation was applied using two $12 \mathrm{~V}$ solenoids (www.me-solve.co.uk) driving a metal rod with a blunt conical tip that contacted participants' skin when a current passed through the solenoids. One solenoid was placed on the tip of the left index finger (i.e. representative tactile locus for the upper limbs); one was placed on the first toe in the left foot (i.e. representative tactile locus for the lower limbs). To mask sounds made by the tactile stimulators, white noise ( $65 \mathrm{~dB}$, measured from the participants' head) was presented through two loudspeakers placed $90 \mathrm{~cm}$ away from the participants' head and $25 \mathrm{~cm}$ to either side of the participants' midline.

During the visual-tactile conditions, trials started with the presentation of a fixation cross $(500 \mathrm{~ms})$, followed by a neutral, angry or sad face $(600 \mathrm{~ms})$. Tactile stimuli were delivered to the left index finger (visual-tactile finger condition, VTFIC) or to the first toe of the left foot (visual-tactile toe condition, VTTOC) $125 \mathrm{~ms}$ after face onset (time point coinciding with the involvement of SCx in visual emotion processing; see Pitcher et al., 2008; Sel et al., 2014). To control for induced visual effects in the somatosensory response, 
we included a visual-only condition (VOC), where the same facial stimuli were presented without tactile stimulation (Fig. 1). Following 180 practice trials that did not contain any experimental material (30 trials per condition, including 10 neutral, 10 angry and 10 sad trials). The overall experiment consisted of 1800 randomized trials, presented in two blocks (900 trials per block/task, including 300 neutral, 300 angry and 300 sad faces).

In $20 \%$ of the trials of each block, participants were asked whether the face stimulus was angry $(10 \%)$ or sad $(10 \%)$. Participants were explicitly told to ignore the tactile stimuli, to closely observe the faces presented on the screen, and to respond vocally (yes/no) as soon as possible if a question was presented (maximum response time $3000 \mathrm{~ms}$ ). This was done to ensure participants directed attention to the task and vocal responses were continuously monitored throughout the task. Participants were encouraged to prevent from blinking during the presentation of the facial stimuli given a break in between blocks. Block order was randomized across participants. Participants were seated in a dimly lit sound-attenuated and electrically shielded chamber in front of a monitor at a distance of $80 \mathrm{~cm}$. Visual stimuli were presented centrally on a black background using E-prime software (Psychology Software Tools, Pittsburgh, PA). Presentation codes and experimental stimuli are available online (https://osf.io/c9bzy).

\subsection{EEG recording and data preprocessing}

EEG was recorded with 60 active electrodes mounted on an elastic cap (two cap sizes, $56 \mathrm{~cm}$ and $58 \mathrm{~cm}$ head circumference) following the $10 \mathrm{M}$ equidistant layout (https://www.easycap.de/wp-content/uploads/2018/02/Easycap-Equidistant-Layouts.pdf).

The distance between electrodes was $3.3 \mathrm{~cm}$ and $3.5 \mathrm{~cm}$ for the $56 \mathrm{~cm}$ and the $58 \mathrm{~cm}$ cap, respectively. Additional 3 electrodes were attached about $1 \mathrm{~cm}$ to the right and left of the eyes and $2 \mathrm{~cm}$ below the left eye for electrooculography recordings (EasyCap GmbH, Herrsching, 
Germany). The Ground electrode was located centrally at the electrode site corresponding to AFz of the 10/20 system. All electrodes were online referenced to the left mastoid and rereferenced to the average reference off-line (average reference included all active electrodes, excluding ocular and non-scalp electrodes). Active electrodes include a circuitry at the electrode site designed to maintain good signal-to-noise ratio promoting good quality of the electrode contact throughout the recording (unlike passive electrodes, active electrodes provide impedance transformation on the electrode). Continuous EEG was recorded using BrainAmp amplifiers (BrainProducts, Munich, Germany; 0.1 $\mu \mathrm{V}$ analog-to-digital conversion resolution; $500 \mathrm{~Hz}$ sampling rate; $0.01-100 \mathrm{~Hz}$ online cut-off filters). Off-line EEG analysis was performed using Vision Analyzer software (BrainProducts, Munich, Germany). The data were digitally low-pass filtered at $40 \mathrm{~Hz}$ and ocular correction was performed (Gratton, Coles, \& Donchin, 1983). When necessary, data were interpolated from neighboring electrodes to replace data from artifactual sites (1-2 electrodes on average). Automatic artifact rejection (i.e. moving window peak-to-peak threshold of $\pm 100 \mu \mathrm{V}$ ) was combined with visual inspection (blind to experimental condition) for all participants. The mean percentage of trials per condition included in the analysis was $88.05 \%$ (S.D.=10.95\%) (percentage of trials did not significantly differ between conditions; $p>0.05$ ). The EEG signal was epoched into $600 \mathrm{~ms}$ segments, starting $100 \mathrm{~ms}$ prior to tactile stimuli onset on VTTOC and VTFIC trials, and starting $25 \mathrm{~ms}$ after visual onset on VOC trials. Segments were then baseline corrected to the first $100 \mathrm{~ms}$.

Single subject ERPs for each condition (VOC, VTFIC, and VTTOC) and emotion (angry, sad, neutral) were calculated and used to compute ERP grand-averages across participants. Specifically, single participant average ERPs were computed for trials in VOC containing only visual evoked potentials (VEPs) and for trials in VTFIC and VTTOC, which contained VEPs and somatosensory-evoked potentials (SEPs). The average number of trials 
for contributing to the VOC, VTFIC, and VTTOC were similar in the neutral $(89.28,88.61$, $88 ; \mathrm{F}=0.56, \mathrm{p}=0.57), \operatorname{sad}(88.83,90.33,90.61 ; \mathrm{F}=1.64, \mathrm{p}=0.21)$ and angry $(90.28,89.56$, 89.17; $\mathrm{F}=0.71, \mathrm{p}=0.40$ ) conditions. To eliminate any contamination of SEPs by VEPs, single subject averages of trials in VOC were subtracted from single subject averages of both VTFIC and VTTOC trials (Arslanova, Galvez-Pol, Calvo-Merino, \& Forster, 2019; Dell'acqua, Jolicoeur, Pesciarelli, Job, \& Palomba, 2003; Sel et al., 2014), and we called this "VEP-free SEPs". The resulting VEP-free SEPs was averaged across participants, and contrasted for angry, sad and neutral.

\subsection{Topography and statistical analysis of VEP-free SEPS}

The topography of the SEPs has a widespread central distribution with greater responses in the hemisphere contralateral to the side of the stimulation. However, in line with the somatotopy organization of the somatosensory cortex, SEPs to tactile stimulation on the finger are mostly represented over the lateral electrode sites, whereas SEPs to tactile stimulation on the toe are typically observed in midline electrodes (Miller, 2012; Shen, Smyk, Meltzoff, \& Marshall, 2018; Xiang et al., 1997). In view of the topographical differences between finger and toe tactile response, we adopted a non-parametric, clusterbased permutation approach to first determine the SEP morphologies in response to tactile stimulation to finger and toe, and then analyze emotion effect on early and mid-latency somatosensory activity. A non-parametric, cluster-based permutation approach is an efficient way of dealing with the multiple comparison problem that prevents biases in selecting timewindows or electrode sites avoiding inflation of type I error rate (Keil et al., 2014; Maris \& Oostenveld, 2007). Mean voltages of the SEPs time-locked to tactile stimulus onset were computed at the group level using a non-parametric randomisation test controlling for multiple-comparisons (Maris \& Oostenveld, 2007). Subject-wise activation time courses 
were passed to analysis procedure of FieldTrip, the details of which are described by Maris and Oostenveld (2007). Subject-wise activation time courses were compared to identify statistically significant spatial and temporal clusters using a FieldTrip-based analysis (Oostenveld, Fries, Maris, \& Schoffelen, 2011). FieldTrip uses a nonparametric method (Bullmore et al., 1999) to address the multiple comparison problem. T-values of adjacent spatial and temporal points whose p-values were less than 0.05 were clustered by adding their t-values, and this cumulative statistic is used for inferential statistics at the cluster level. This procedure, that is, the calculation of t-values at each spatial and temporal point followed by clustering of adjacent t-values was repeated 5000 times, with randomised swapping and resampling of the subject-wise averages before each repetition. This Monte Carlo method results in a nonparametric estimate of the P-value representing the statistical significance of the identified cluster.

The topographical distribution of the neural phenomena comprising the SEP responses to finger and toe tactile stimulation was defined as following. We first computed mean voltages of the SEPs time-locked to tactile stimulus onset for all finger trials and all toe trials, separately. The morphology analysis was done at the group level with a non-parametric one-sample randomization test including all electrodes sites and across the entire time window (i.e. 0 - 500ms after stimuli onset) and separately for finger and toe trials. For these analyses, no a-priori electrode clusters were formed (i.e. all active electrodes were treated as a distinct variable). The topography analysis revealed a series of time windows overlapping with previously reported early and mid-latency somatosensory responses (Forster \& Eimer, 2005) as well as a number of electrode sites spread along the centro-frontal and centroparietal areas that were different for finger vs. toe tactile responses. These electrodes were then organized in regions of interest (ROIs) according to their spatial distribution (Fig. 2) for 
further processing. To avoid spurious findings, significant effects of $15 \mathrm{~ms}$ or shorter were discarded from further analysis.

To test if the somatosensory cortex shows discrete somatotopic activations when observing others' facial expressions, we first computed the emotion effect on finger and toe VEP-free SEPs (calculated by subtraction of amplitudes at each time point of the neutral trials from the angry and the sad trials). We then contrasted the anger $v s$. sadness emotion effects separately for finger and toe SEPs by means of non-parametric cluster-based permutation test. To estimate the emotion effects on neural responses to finger and toe tactile stimulation the voltages of the SEPs comprised in the time windows resulting from the morphology analysis were selected. Likewise, analyses were restricted to the ROIs (Fig. 2) defined according to the finger and toe SEP morphology analysis. For each time window, subject-wise activations at electrode sites circumscribed in every ROI were extracted and passed to the analysis procedure. Where appropriate, $\mathrm{p}$-values were corrected for multiple comparisons using Bonferroni-Holms correction.

\subsection{Topography and statistical analysis of VEPs}

Additionally, to ensure that the emotion manipulation was effective, mean voltages of the VEPs time-locked to face onset in the VOC were analyzed mimicking the procedure followed in the SEPs analysis. In brief, we first performed a topography analysis computing the subject-wise activation time courses, for all trial types at the group level using the nonparametric randomization test at all electrode sites, and across the time window ranging from 0 to $500 \mathrm{~ms}$. The topography analysis revealed a series of time windows where early and long-latency VEP components have been typically reported (Williams et al., 2004, 2006; Conty et al., 2012), and a number of electrode sites in fronto-central, central, centro-parietal and temporoparietal areas. These electrodes sites were organized in ROIs according to their 
spatial distribution. We then tested the emotion effects (computed by the difference between neutral and angry trials, and the difference between neutral and sad trials) at the selected time windows and ROIs following the morphology analysis. Subject-wise activations at the chosen times and electrode sites were extracted and passed to the non-parametric randomization analysis correcting for multiple comparisons.

Furthermore, to rule out that the responses of SCx to observed emotional expressions were totally independent from emotional visual responses, we perform a correlation between the existing amplitude changes in somatotopic responses and the VEP modulations to emotions. A lack of correlation between these two measurements would corroborate their independence.

\subsection{Emotional ratings}

We explored the idea of whether the changes observed in finger and toe VEP-free SEPs might be associated with participants' subjective reports of changes in bodily sensations to experiencing emotions. Thus, at the end of the EEG session participants were presented again with the same facial expressions observed during the EEG session. These expressions were presented in separate trials along with two 2D body silhouettes (excluding head, Supplementary Fig.). Following the instructions reported in Nummenmaa et al (2014) volunteers were asked to indicate the bodily location where the person in the picture was experiencing most - silhouette A - or least - silhouette B - the expressed facial emotion. Volunteers responded using the two 2D body silhouettes presented on each side of the facial expression (Supplementary Fig.) and, unlike Nummenmaa et al (2014), their responses were restricted to a single mouse click in a given location within the silhouettes (presentation code is available online https://osf.io/c9bzy). These results were correlated with the observed emotion effects on VEP-free SEPs (see Supplementary material for details). EEG and 
behavioural data are not currently available as the ethics used in the current study did not include explicit approval from Research Ethics Committee, Psychology Department, City University London to share the data in a public repository. Data can be made available upon request and in the condition that requestors are willing to accept the data sharing agreement from the Research Ethics Committee, Psychology Department, City University London. The preprocessing analysis code built in Brain Vision Analyzer can only be opened together with raw data. Therefore, this code can be made available together with the raw data. The code used for statistical analysis is available online (https://osf.io/c9bzy).

\section{Results}

\subsection{VEP-free SEPs topography results}

In line with previous investigations showing different topographical distribution of somatosensory responses to finger $v s$. toe tactile stimulation, the morphology analysis revealed that the finger VEP-free SEPs expressed distinct topographical distribution in comparison to the toe VEP-free SEPs over the fronto-central and centro-parietal sites located close to and over somatosensory cortex (Forster \& Eimer, 2005; Sel et al., 2014; Xiang et al., 1997). While the finger VEP-free SEPs were distributed over sites comprising lateral and middle sites, the toe SEPs were mostly distributed over middle sites. Specifically, the topography results of the cluster-based permutation analysis showed that finger VEP-free SEPs significantly differed from zero in the following centro-lateral sites: $\mathrm{Cz}, \mathrm{FCz}, \mathrm{C} 2, \mathrm{CP} 2$, CPz, CP1, C1, F2, FC4, C4, CP4, P2, Pz, P1, CP3, PO4, PO3 (p = 0.001; 156-278ms; negative cluster group). Moreover, the topography results of the cluster-based permutation analysis showed that toe VEP-free SEPs significantly differed from zero in the following middle electrode sites: Cz, FCz, C2, CP2, CPz, CP1, C1, Fz, F2, P1, CP3, C3, FC3, F1 (p = 0.001; 172-310ms; negative cluster). Accordingly, these electrode sites in centro-lateral and middle areas were organized in spatial regions (Fig. 2). 


\subsection{Emotion-specific modulation of VEP-free SEP amplitudes}

The results of the cluster-based permutation analysis revealed significant differences when contrasting the angry $(M=-0.134 \mu \mathrm{V} ; S D=0.948) v s$. $\operatorname{sad}(M=0.195 \mu \mathrm{V} ; S D=0.647)$ emotion effects on finger VEP-free SEPs in the 224-258ms time window in the right mediodorsal ROI (CP2, C4, CP4; $\mathrm{p}=0.008$; cluster stat $=50.93)($ Fig. 2). These electrode sites are located close to and over somatosensory cortex and the time window coincide with the latency of mid early SEP latency (Forster \& Eimer, 2005) (there were not significant differences between anger $(M=-0.144 \mu \mathrm{V} ; S D=0.930) v s$. sadness $(M=-0.025 \mu \mathrm{V} ; S D=0.787)$ effects on finger VEP-free SEPs at any other cluster electrode). Contrary, the anger effects did not significantly differ from the sadness effects on the toe VEP-free SEPs at any electrode cluster, or in any other cluster electrode on finger VEP-free SEPs.

\subsection{VEPs topography results}

To ensure that the emotion manipulation was effective, we contrasted the effects of anger vs. sadness on VEPs time-locked to face onset in the visual only condition. In line with the SEP analysis, we first performed a topography analysis on the average signal across conditions (i.e. neutral, angry, sad) to characterize the temporal and spatial distribution of the VEPs to facial expressions. The results of the topography analysis revealed that the VEP responses were significantly different to zero in two positive clusters. The first positive cluster $(\mathrm{p}=0.001)$ ranging from 210 to $350 \mathrm{~ms}$ after face onset included the following electrodes: Cz, FCz, C2, CP2, CPz, CP1, C1, Fz, F2, FC4, C4, C3, FC3, F1, AF4, F6, FC6, C6, C5, FC5, F5, AF3, F8, FT8, T8, TP8, TP7, T7, FT7, F7, F10, FT10, TP10, TP9, FT9, F9. The second positive cluster $(\mathrm{p}=0.003$ ) observed from 0 to $164 \mathrm{~ms}$ after stimuli onset comprised the following electrodes: $\mathrm{Cz}, \mathrm{FCz}, \mathrm{C} 2, \mathrm{CP} 2, \mathrm{CPz}, \mathrm{CP} 1, \mathrm{C} 1, \mathrm{Fz}, \mathrm{F} 2, \mathrm{FC} 4, \mathrm{C} 4, \mathrm{CP} 4$, 
P2, Pz, P1, C3, FC3, F1, AF4, F6, FC6, C6, PO4, PO3, P3, C1, F5, AF3, F8, FT8, T8, Oz, O1, T7, FT7, F7, CP1, FC5. Electrode sites from these two clusters were organized in ROIs according to their spatial distribution (Frontocentral ROI: Cz, FCz, C2, CP2, CP1, C1, Fz, F2, FC4, C4, C3, FC3, F1, AF4, F6, F5, AF3; Lateral ROI: FC6, C6, C5, FC5, F8, FT8, T8, T7, FT7, F7, F10, FT10, FT9, F9; Temporal ROI: TP9, TP7, TP10, TP9; Centroparietal ROI: CPz, CP4, P2, PZ, P1, CP3; Parieto-occipital ROI: PO4, PO3, P3, OZ, O1) before performing the cluster-based permutation analysis to investigate valence-specific effects on VEPs.

\subsection{Emotion-specific modulation of VEP amplitudes}

The results of the cluster-based permutation analysis contrasting the angry vs. sad emotion effects on VEPs revealed significant differences between the conditions of interest in two negative clusters. One in frontocental sites $(\mathrm{p}=0.009)$ between $210-280 \mathrm{~ms}$ after face onset; one in centroparietal sites $(\mathrm{p}=0.002)$ between $210-396 \mathrm{~ms}$. In addition, the analysis revealed a temporal positive cluster at $210-358 \mathrm{~ms}$ latency $(\mathrm{p}=0.001)$. These latencies and electrode sites overlap with previously reported valence specific emotional effects on early and mid-latency cortical responses to facial expression, namely N250 and P300 - see Fig. 3 (Balconi \& Pozzoli, 2003; Conty, Dezecache, Hugueville, \& Grèzes, 2012; Schupp 2004; Williams et al., 2004; Williams, Palmer, Liddell, Song, \& Gordon, 2006). These results accord with previous observations of early and mid-latency VEP modulation in response to direct attention to faces, and confirms the effectiveness of the visual manipulation. In addition, there was a lack of correlation between emotion modulation in VEPs and amplitude changes in SCx to observed emotions (all ps > 0.05), confirming that the somatosensory responses to emotional faces are independent, over and above, visual processing.

Overall, these results show that observing others' emotional expressions, namely anger $v s$ sadness, leads to emotion-specific somatotopic activations in the hand area of the 
somatosensory cortex. We observed a rapid, emotion-specific sensitivity whereby finger somatosensory activity differentially contributes to the observation of angry $v s$ sad facial expressions. By contrast, we did not observe emotion-specific effects on somatosensory toe activation when observing angry $v s$ neutral faces. In addition, we observed valence-specific effects of anger $v s$. sadness on VEPs time-locked to the face onset which support the effectiveness of the emotional manipulation.

\section{Discussion}

The present study investigated whether the SCx exhibits discrete somatotopic responses to the observation of distinctive emotional facial expressions. The pattern of results observed in probed SCx responses indicate that, in contrast to observing neutral faces, observing angry facial expressions leads to a decreased response in the finger somatosensory cortex, whereas the opposite pattern of finger somatotopic activation emerged when observing sad faces. In other words, finger SCx responses to anger differed from SCx responses to neutral faces to a degree that was significantly different than when contrasting SCx to sad $v s$ neutral faces. These effects were observed in pure SEP responses. This is, by subtracting VEP responses from SEP response we controlled for the influence of any carryover effects from visual processing on tactile responses. By contrast, these emotion-specific activations were not observed in toe somatotopic activity. Toe SCx responses to anger differed from SCx responses to neutral faces in a similar degree than SCx responses to sadness differed from SCx responses to neutral expressions. These findings demonstrate that distinctive emotional expressions differentially activate somatotopic representations of the body-parts linked to the subjective experience of others' facial emotions.

The contribution of the SCx during emotional face observation has been proven crucial and independent from parallel visual processing, allowing an internal embodied 
representation of the observed emotion (Pitcher et al., 2008; Pourtois et al., 2004; Sel et al., 2014). The SCx exhibits responses that are somatotopic in nature. Thus, touch to the right hand as well as observing others' actions performed with the right hand, leads to activations in the left SCx over the hand area as opposed to activations in other bodily areas within the SCx (Avikainen, Forss, \& Hari, 2002; Rossi et al., 2002). In this line, the current results show that the SCx exhibits distinctive somatotopic activations to different facial emotional expressions in two different bodily parts. In specifics, while finger SCx distinctively responds to angry $v s$. sadness, similar pattern of activations for the two emotions were not found in toe SCx. Our results extend previous findings suggesting that, beyond the sensory somatotopy to touch and observed movements, the perception of emotional facial expressions rely on somatotopic activations in SCx that are unique to the observed emotion, in this case anger vs sadness. These results extend previous neuroimaging findings, providing evidence for a direct link between somatotopic activations and observed emotions.

The role of the body in emotion has been revisited recently in a series of investigations. These studies suggest that, rather than an overall engagement of the body to the subjective experience of emotions, specific patterns of activations including distinctive sets of bodily parts underpin the experience of individual emotions (Nummenmaa et al., 2014; Nummenmaa et al., 2018; Volynets et al., In press). Thus, for example the feeling of anger and sadness, both engage a number of areas in the upper body, including the hand, and they do so in an opposite manner, i.e. activity increase $v s$. decrease for angry as opposed to sad feelings, respectively. Also, the results from the bodily maps of emotions reported by Nummenmaa and cols (2014) reveal that in the feet area, participants reported a significant activation decrease of around -15 points on a scale ranging from 15 to -15 points. However, the subjective reports for anger indicate a moderate activation increase in the feet of around 5 points (Nummenmaa et al., 2014; Volynets et al., In press). In this line, by directly tapping 
with tactile probes into representative sites of upper (finger) and lower (toe) body sites, we demonstrated opposed finger SCx responses to sadness $v s$. anger, and lack of difference between these emotions in toe SCx response. Direct comparison between modulation of somatosensory amplitude responses to emotions in the current study, and subjective changes in bodily activations reported previously is proven challenging because of the obvious methodological differences. However, neuroimaging findings have suggested that emotions related to action tendencies, particularly highly salient angry stimuli (Frijda, Kuipers, \& ter Schure, 1989; Harmon-Jones, Harmon-Jones, \& Price, 2013) engage activation of somatomotor areas and areas adjacent to the $\mathrm{SCx}$, together with areas in the limbic system that generally respond to all emotions (Saarimäki et al., 2015). Therefore, one could argue that the amplitude differences observed in finger VEP-free SEPs between anger and sadness are linked to a greater engagement of the in finger $\mathrm{SCx}$ driven by higher action tendencies to angry vs. sad faces (Hammer \& Marsh, 2015). Particularly, it is possible that the greater SCx engagement to anger is expressed in activity reduction to observed facial expressions in form of sensory resonance (Moore, Gorodnitsky, \& Pineda, 2012). Moreover, the lack of difference between anger and sadness in toe VEP-free SEPs could be explained by a lesser engagement towards action of this bodily area to the experience of sadness - i.e. the bodily map of emotions suggests an activation decrease in the lower limbs to sadness, whereas activation increase in this area is not so obvious to anger. This pattern of bodily activation for anger vs sadness is partially in line with the current findings. This hypothesis would need further testing by systematically comparing SCx responses from different bodily parts to observation of a range of high-arousing (e.g. happiness, fear, anger) and low-arousing (sadness, disgust) emotions.

Topographic responses of emotions exist in other sensory domains such as vision. Thus, neurons in face-selective regions such as the face fusiform area (FFA) and the superior 
temporal sulcus (STS) show emotion-specific representations distinguishing between positive, negative and neutral facial expressions (Engell \& Haxby, 2007; Said, Moore, Engell, Todorov, \& Haxby, 2010; Skerry \& Saxe, 2014). Similarly, electrophysiological responses recorded from visual cortices exhibit distinctive amplitude changes to facial expressions that differ in their emotional valence (Williams et al., 2006). For example, threatening angry stimuli lead to enhanced visual responses from around $200 \mathrm{~ms}$ after stimuli onset (Conty et al., 2012), whereas these amplitude changes in mid-to-late visual evoked responses are less obvious to low-arousal expressions like sadness (Balconi \& Pozzoli, 2003; Schupp 2004). Our results replicate previous emotional effects on early ERPs, demonstrating a greater modulation for angry $v s$. sad expressions in mid-latency visual evoked responses both in parietal and frontocentral areas. This pattern of results mimics the emotion-specific activations in SCx, and confirms the efficacy of the visual manipulation. Importantly, while both somatosensory and visual cortices exhibit similar parallel patterns of activations to angry $v s$. sadness, SCx modulations are entirely independent, over and above the emotionrelated changes in visual cortex as enabled by the ERP subtraction method and proven by the lack of correlation between the emotion effects on somatosensory and visual cortical responses. Our results extend previous investigations on emotion-specific modulations in visual cortex (Balconi \& Pozzoli, 2003; Conty et al., 2012; Schupp 2004; Williams et al., 2004; Williams et al., 2006), favouring the idea that sensory representations of emotions are supramodal (Peelen, Atkinson, \& Vuilleumier, 2010)

A number of investigations have proven the efficacy of the ERP subtraction method to isolate somatosensory responses from visual processing (Arslanova et al., 2019; GalvezPol, Calvo-Merino, Capilla, \& Forster, 2018; Galvez-Pol, Forster, \& Calvo-Merino, 2018; Sel et al., 2014). For example, Sel and colleagues (2014) showed independent SCx responses to highly salient happy and fearful faces, as opposed to neutral faces, supporting the idea that 
highly salient approach emotions recruit sensorimotor cortices to a greater extent than neutral faces (Saarimäki et al., 2015). Interestingly, they reported similar activations in both face and finger somatotopic representations comparably to the subjective maps of emotions (Nummenmaa et al., 2014; Nummenmaa et al., 2018), whereby both happiness and fear are linked to increase activation in the upper body including face and arms. Moreover, the direct contrast between the former (i.e. Sel et al., 2014) and the current study reveals emotionspecific modulations of somatosensory activity at early- $v s$. mid-latencies to observation of happiness/fear and anger/sadness, respectively. These results are in parallel to investigations in the visual domain demonstrating that distinctive emotions modulate cortical responses at different latencies - for instance, fearful faces lead to amplitude changes starting $170 \mathrm{~ms}$ after stimuli onset, whereas sad faces are associated with changes in the mid-latencies from around 250ms after stimuli onset (Balconi \& Pozzoli, 2003; Schupp 2004; Williams et al., 2004; Williams et al., 2006). Overall, across a number of studies we have demonstrated that the somatosensory cortex independently contributes to the understanding of observed emotions, and it does it so, in a somatotopic manner.

The current study has a number of limitations that are worth noting. First, our approach to investigate somatotopic engagement to visual processing is limited to tactile stimulation of discrete bodily parts, i.e. finger, face or toe. In order to perform a systematic investigation of the contribution of each bodily part to discrete emotions, we would need to extend this protocol by applying tactile stimulation to a define set of bodily areas, including arms, chest and torso, and legs. Equally, these examinations would require including other basic emotions such as disgust, as well as, non-basic emotions such as anxiety, love, depression or pride. Future studies should investigate whether the somatotopic responses to facial emotional expression extend to other emotional domains, such as emotional bodily expressions, or other sensory modalities such as music or emotional sounds. This approach 
would contribute to reveal the somatotopic neural dynamics to others' emotions. In addition, we did not find a relationship between emotion-related changes in VEP-free somatosensory responses and self-reported bodily sensations in upper/lower limbs. This could be due to the way the self-reported data was collected. Participants used one single mouse click to indicate where the observed person felt the expressed emotion. This approach contrasts with previous studies where participants were allowed to freely draw on a mannequin (Nummenmaa et al., 2014), i.e. the current task might have been less sensitive than previously reported tasks. In addition, we investigated bodily changes to observed facial expressions, whereas previous studies used a variety of emotional stimuli, including words, stories, movies, etc. This limitation should be addressed in future studies adopting a more sensitive behavioural measure and a variety of emotional stimuli to examine subjective emotions.

\section{Conclusions}

To conclude, the current study suggests a distinctive role of the SCx in emotional processing, allowing us to represent others' emotional feelings by rapid and visuallyindependent somatotopic activations. The data reveals that representing others' emotions is instantiated through the cortical representations of one's body-parts previously linked to the subjective experience of bodily sensations, providing a potential neural correlate for somatotopic emotion understanding. These findings advance simulation theories suggesting a close linkage between the others' body-sensations and the observer's body, in particular, a body site-specific representation response during the observation of emotional (angry/sad) vs. neutral faces. In essences, SCx allows to represent others' emotions as we "feel" them in the body. 
Declarations of interest: none

Acknowledgments: Work supported by the European Research Council (ERC-2010-StG262853) to MT, by the Spanish Ministry of Economy and Competitiveness (MINECO/RYC2008-03090 and PSI2012-34558) to BC-M, and by the Bial Foundation (Grant 44/16) to AS. 


\section{References}

Arslanova, I., Galvez-Pol, A., Calvo-Merino, B., \& Forster, B. (2019). Searching for bodies: ERP evidence for independent somatosensory processing during visual search for body-related information. NeuroImage, 195, 140-149. doi:https://doi.org/10.1016/j.neuroimage.2019.03.037

Atkinson, A. P., \& Adolphs, R. (2011). The neuropsychology of face perception: beyond simple dissociations and functional selectivity. Philos Trans R Soc Lond B Biol Sci, 366(1571), 1726-1738. doi:10.1098/rstb.2010.0349

Avikainen, S., Forss, N., \& Hari, R. (2002). Modulated Activation of the Human SI and SII Cortices during Observation of Hand Actions. NeuroImage, 15(3), 640-646. doi:https://doi.org/10.1006/nimg.2001.1029

Balconi, M., \& Pozzoli, U. (2003). Face-selective processing and the effect of pleasant and unpleasant emotional expressions on ERP correlates. International Journal of Psychophysiology, 49(1), 67-74. doi:https://doi.org/10.1016/S0167-8760(03)00081-3

Barrett, L. F. (2006). Are Emotions Natural Kinds? Perspectives on Psychological Science, 1(1), 28-58. doi:10.1111/j.1745-6916.2006.00003.x

Baseler, H. A., Harris, R. J., Young, A. W., \& Andrews, T. J. (2012). Neural Responses to Expression and Gaze in the Posterior Superior Temporal Sulcus Interact with Facial Identity. Cerebral Cortex, 24(3), 737-744. doi:10.1093/cercor/bhs360

Bastiaansen, J. A. C. J., Thioux, M., \& Keysers, C. (2009). Evidence for mirror systems in emotions. Philosophical Transactions of the Royal Society B: Biological Sciences, 364(1528), 2391-2404. doi:doi:10.1098/rstb.2009.0058

Bullmore, E. T., Suckling, J., Overmeyer, S., Rabe-Hesketh, S., Taylor, E., \& Brammer, M. J. (1999). Global, voxel, and cluster tests, by theory and permutation, for a difference 
between two groups of structural MR images of the brain. IEEE Transactions on Medical Imaging, 18(1), 32-42. doi:10.1109/42.750253

Carr, L., Iacoboni, M., Dubeau, M.-C., Mazziotta, J. C., \& Lenzi, G. L. (2003). Neural mechanisms of empathy in humans: A relay from neural systems for imitation to limbic areas. Proceedings of the National Academy of Sciences, 100(9), 5497-5502. doi:10.1073/pnas.0935845100

Conty, L., Dezecache, G., Hugueville, L., \& Grèzes, J. (2012). Early Binding of Gaze, Gesture, and Emotion: Neural Time Course and Correlates. The Journal of Neuroscience, 32(13), 4531-4539. doi:10.1523/jneurosci.5636-11.2012

Damasio Antonio, R., Everitt Barry, J., Bishop, D., Roberts, A. C., Robbins Trevor, W., \& Weiskrantz, L. (1996). The somatic marker hypothesis and the possible functions of the prefrontal cortex. Philosophical Transactions of the Royal Society of London. Series B: Biological Sciences, 351(1346), 1413-1420. doi:10.1098/rstb.1996.0125

Dell'acqua, R., Jolicoeur, P., Pesciarelli, F., Job, R., \& Palomba, D. (2003). Electrophysiological evidence of visual encoding deficits in a cross-modal attentional blink paradigm. Psychophysiology, 40(4), 629-639. doi:10.1111/1469-8986.00064

Engell, A. D., \& Haxby, J. V. (2007). Facial expression and gaze-direction in human superior temporal sulcus. Neuropsychologia, 45(14), 3234-3241. doi:http://dx.doi.org/10.1016/j.neuropsychologia.2007.06.022

Forster, B., \& Eimer, M. (2005). Covert attention in touch: Behavioral and ERP evidence for costs and benefits. Psychophysiology, 42(2), 171-179. doi:10.1111/j.14698986.2005.00268.x

Frijda, N. H., Kuipers, P., \& ter Schure, E. (1989). Relations among emotion, appraisal, and emotional action readiness. Journal of personality and social psychology, 57(2), 16. doi:doi:10.1037/0022-3514.57.2.212 
Galvez-Pol, A., Calvo-Merino, B., Capilla, A., \& Forster, B. (2018). Persistent recruitment of somatosensory cortex during active maintenance of hand images in working memory. NeuroImage, 174, 153-163. doi:https://doi.org/10.1016/j.neuroimage.2018.03.024

Galvez-Pol, A., Forster, B., \& Calvo-Merino, B. (2018). Modulation of motor cortex activity in a visual working memory task of hand images. Neuropsychologia, 117, 75-83. doi:https://doi.org/10.1016/j.neuropsychologia.2018.05.005

Galvez-Pol, A., Calvo-Merino, B., \& Forster, B. (2020). Revealing the body in the brain: an ERP method to examine sensorimotor activity during visual perception of bodyrelated information. Cortex. doi:https://doi.org/10.1016/j.cortex.2020.01.017

Gratton, G., Coles, M. G., \& Donchin, E. (1983). A new method for off-line removal of ocular artifact. Electroencephalography and clinical neurophysiology, 55(4), 468484.

Hammer, J. L., \& Marsh, A. A. (2015). Why do fearful facial expressions elicit behavioral approach? Evidence from a combined approach-avoidance implicit association test. Emotion (Washington, D.C.), 15(2), 223-231. doi:10.1037/emo0000054

Harmon-Jones, E., Harmon-Jones, C., \& Price, T. F. (2013). What is Approach Motivation? Emotion Review, 5(3), 291-295. doi:10.1177/1754073913477509

Hess, U., \& Fischer, A. (2014). Emotional Mimicry: Why and When We Mimic Emotions. Social and Personality Psychology Compass, 8(2), 45-57. doi:10.1111/spc3.12083 Keil, A., Debener, S., Gratton, G., Junghöfer, M., Kappenman, E. S., Luck, S. J., . . Yee, C. M. (2014). Committee report: Publication guidelines and recommendations for studies using electroencephalography and magnetoencephalography. Psychophysiology, 51(1), 1-21. doi:10.1111/psyp.12147

Keysers, C., Kaas, J. H., \& Gazzola, V. (2010). Somatosensation in social perception. Nature Reviews Neuroscience, 11, 417. doi:10.1038/nrn2833 
Lange. C.G. The mechanisms of the emotions. B. Rand (Ed.), The Classical Psychologists, Houghton Mifflin, Boston (1885/1912), pp. 672-684 (Classics Editor's note: This translation of a passage from Lange's Om Sindsbevaegelser (1885) from Lange's Ueber Gemüthsbewegungen. Eine psycho-physiologische Studie (1887))

Levenson, R. W. (2003). Blood, sweat, and fears: The autonomic architecture of emotion. Annals of the New York Academy of Sciences, 1000(1), 348-366.

Lundqvist, D., Flykt, A., \& Öhman, A. (1998). The Karolinska directed emotional faces (KDEF). CD ROM from Department of Clinical Neuroscience, Psychology section, Karolinska Institutet, 91, 630.

Maris, E., \& Oostenveld, R. (2007). Nonparametric statistical testing of EEG- and MEG-data. Journal of Neuroscience Methods, 164(1), 177-190. doi:https://doi.org/10.1016/j.jneumeth.2007.03.024

Miller, J. (2012). Selection and preparation of hand and foot movements: Cz activity as a marker of limb system preparation. Psychophysiology, 49(5), 590-603. doi:10.1111/j.1469-8986.2011.01338.x

Moore, A., Gorodnitsky, I., \& Pineda, J. (2012). EEG mu component responses to viewing emotional faces. Behavioural Brain Research, 226(1), 309-316. doi:https://doi.org/10.1016/j.bbr.2011.07.048

Montoya, P., \& Sitges, C. (2006). Affective modulation of somatosensory-evoked potentials elicited by tactile stimulation. Brain Research, 1068(1), 205-212. doi:https://doi.org/10.1016/j.brainres.2005.11.019

Moore, A., Gorodnitsky, I., \& Pineda, J. (2012). EEG mu component responses to viewing emotional faces. Behavioural Brain Research, 226(1), 309-316. doi:https://doi.org/10.1016/j.bbr.2011.07.048 
Nakamura, K., Kawashima, R., Ito, K., Sugiura, M., Kato, T., Nakamura, A., . . Kojima, S. (1999). Activation of the Right Inferior Frontal Cortex During Assessment of Facial Emotion. Journal of Neurophysiology, 82(3), 1610-1614. doi:10.1152/jn.1999.82.3.1610

Niedenthal, P. M. (2007). Embodying Emotion. Science, 316(5827), 1002-1005. doi:10.1126/science. 1136930

Nummenmaa, L., Glerean, E., Hari, R., \& Hietanen, J. K. (2014). Bodily maps of emotions. Proceedings of the National Academy of Sciences, 111(2), 646-651. doi:10.1073/pnas.1321664111

Nummenmaa, L., Hari, R., Hietanen, J. K., \& Glerean, E. (2018). Maps of subjective feelings. Proceedings of the National Academy of Sciences, 115(37), 9198-9203. doi:10.1073/pnas.1807390115

Nummenmaa, L., Hirvonen, J., Parkkola, R., \& Hietanen, J. K. (2008). Is emotional contagion special? An fMRI study on neural systems for affective and cognitive empathy. NeuroImage, 43(3), 571-580. doi:https://doi.org/10.1016/j.neuroimage.2008.08.014

Oldfield, R. C. (1971). The assessment and analysis of handedness: The Edinburgh inventory. Neuropsychologia, 9(1), 97-113. doi:https://doi.org/10.1016/0028-3932(71)90067-4

Oostenveld, R., Fries, P., Maris, E., \& Schoffelen, J.-M. (2011). FieldTrip: Open Source Software for Advanced Analysis of MEG, EEG, and Invasive Electrophysiological Data. Computational Intelligence and Neuroscience, 2011, 156869. doi:10.1155/2011/156869

Peelen, M. V., Atkinson, A. P., \& Vuilleumier, P. (2010). Supramodal Representations of Perceived Emotions in the Human Brain. The Journal of Neuroscience, 30(30), 10127-10134. doi:10.1523/jneurosci.2161-10.2010 
Pitcher, D., Garrido, L., Walsh, V., \& Duchaine, B. C. (2008). Transcranial Magnetic Stimulation Disrupts the Perception and Embodiment of Facial Expressions. The Journal of Neuroscience, 28(36), 8929-8933. doi:10.1523/jneurosci.1450-08.2008

Pourtois, G., Sander, D., Andres, M., Grandjean, D., Reveret, L., Olivier, E., \& Vuilleumier, P. (2004). Dissociable roles of the human somatosensory and superior temporal cortices for processing social face signals. European Journal of Neuroscience, 20(12), 3507-3515. doi:10.1111/j.1460-9568.2004.03794.x

Ravaja, N., Harjunen, V., Ahmed, I., Jacucci, G., \& Spapé, M. M. (2017). Feeling Touched: Emotional Modulation of Somatosensory Potentials to Interpersonal Touch. Scientific Reports, 7, 40504-40504. doi:10.1038/srep40504

Rossi, S., Tecchio, F., Pasqualetti, P., Ulivelli, M., Pizzella, V., Romani, G. L., . . Rossini, P. M. (2002). Somatosensory processing during movement observation in humans. Clinical Neurophysiology, 113(1), 16-24. doi:https://doi.org/10.1016/S13882457(01)00725-8

Saarimäki, H., Ejtehadian, L. F., Glerean, E., Jääskeläinen, I. P., Vuilleumier, P., Sams, M., \& Nummenmaa, L. (2018). Distributed affective space represents multiple emotion categories across the human brain. Social Cognitive and Affective Neuroscience, 13(5), 471-482. doi:10.1093/scan/nsy018

Saarimäki, H., Gotsopoulos, A., Jääskeläinen, I. P., Lampinen, J., Vuilleumier, P., Hari, R., . . . Nummenmaa, L. (2015). Discrete Neural Signatures of Basic Emotions. Cerebral Cortex, 26(6), 2563-2573. doi:10.1093/cercor/bhv086

Said, C. P., Moore, C. D., Engell, A. D., Todorov, A., \& Haxby, J. V. (2010). Distributed representations of dynamic facial expressions in the superior temporal sulcus. Journal of vision, 10(5), 11-11. doi:10.1167/10.5.11 
Schupp , H. T., A. Öhman, M. Junghöfer, A.I. Weike, J. Stockburger, A.O. Hamm. (2004).

The facilitated processing of threatening faces: An ERP analysis. Emotion (Washington, D.C.), 4 (2), 189.

Sel, A., Forster, B., \& Calvo-Merino, B. (2014). The emotional homunculus: ERP evidence for independent somatosensory responses during facial emotional processing. Journal of Neuroscience, 34(9), 3263-3267.

Shen, G., Smyk, N. J., Meltzoff, A. N., \& Marshall, P. J. (2018). Using somatosensory mismatch responses as a window into somatotopic processing of tactile stimulation. Psychophysiology, 55(5), e13030. doi:10.1111/psyp.13030

Skerry, A. E., \& Saxe, R. (2014). A common neural code for perceived and inferred emotion. The Journal of neuroscience : the official journal of the Society for Neuroscience, 34(48), 15997-16008. doi:10.1523/JNEUROSCI.1676-14.2014

Torregrossa, L. J., Snodgress, M. A., Hong, S. J., Nichols, H. S., Glerean, E., Nummenmaa, L., \& Park, S. (2018). Anomalous Bodily Maps of Emotions in Schizophrenia. Schizophrenia Bulletin. doi:10.1093/schbul/sby 179

Volynets, S., Glerean, E., Hietanen, J. K., Hari, R., \& Nummenmaa, L. (In press). Bodily maps of emotions are culturally universal. Emotion. Emotion (Washington, D.C.).

Vuilleumier, P., \& Pourtois, G. (2007). Distributed and interactive brain mechanisms during emotion face perception: evidence from functional neuroimaging. Neuropsychologia, 45(1), 174-194.

Williams, L. M., Liddell, B. J., Rathjen, J., Brown, K. J., Gray, J., Phillips, M., . . Gordon, E. (2004). Mapping the time course of nonconscious and conscious perception of fear: An integration of central and peripheral measures. Human Brain Mapping, 21(2), 6474. doi:10.1002/hbm.10154 
Williams, L. M., Palmer, D., Liddell, B. J., Song, L., \& Gordon, E. (2006). The ‘when’ and 'where' of perceiving signals of threat versus non-threat. NeuroImage, 31(1), 458467. doi:https://doi.org/10.1016/j.neuroimage.2005.12.009

Xiang, J., Kakigi, R., Hoshiyama, M., Kaneoke, Y., Naka, D., Takeshima, Y., \& Koyama, S. (1997). Somatosensory evoked magnetic fields and potentials following passive toe movement in humans. Electroencephalography and Clinical Neurophysiology/Evoked Potentials Section, 104(5), 393-401. doi:https://doi.org/10.1016/S01685597(97)00058-0 


\section{Caption to figures}

Figure 1: Timeline of visual-tactile toe condition (VTTOC), visual-tactile finger condition (VTFIC) and visual only condition (VOC) in the emotion task. In VOC faces were presented alone. In VTTOC and VTFIC, tactile probes were delivered $125 \mathrm{~ms}$ after the face onset to the toe and the finger, respectively. In both tasks, on $20 \%$ of trials participants were asked to indicate the emotional content of the stimulus after presentation of the face

Figure 2: Grand average difference somatosensory-evoked activity (VEP-free SEPs) when observing angry (red lines), sad (blue lines) and neutral (black lines) faces, for electrodes where differences were strongest (marked in the maps adjacent to the graphs) in the VTTOC (right graph) and in the VTFIC (left graph) condition (negative polarity down). The inset to each graph shows the average amplitude and standard error of the mean of emotion effects (angry - neutral; sad - neutral) for the 224-258ms time window, where the difference between emotion effects in the finger area were significant. Topographical maps showing enhanced somatosensory activity when observing angry, neutral and sad faces for the finger (left) and the toe (right) tactile condition, respectively (electrode montage- 60 channels, average reference).

Figure 3: Grand average VEPs evoked by the onset of the face images when angry (red lines), sad (blue lines) and neutral (black lines) faces at frontocentral (Cz, FCz, C2, CP2, CP1, C1, Fz, F2, FC4, C4, C3, FC3, F1, AF4, F6, F5, AF3), centroparietal (CPz, CP4, P2, PZ, P1, CP3) and temporal (TP9, TP7, TP10, TP9) electrode positions for which maximum amplitude differences at N200, P230, N250 and P300 time-windows were observed (negative polarity down; electrode montage- 60 channels, average reference). 
Figure 1

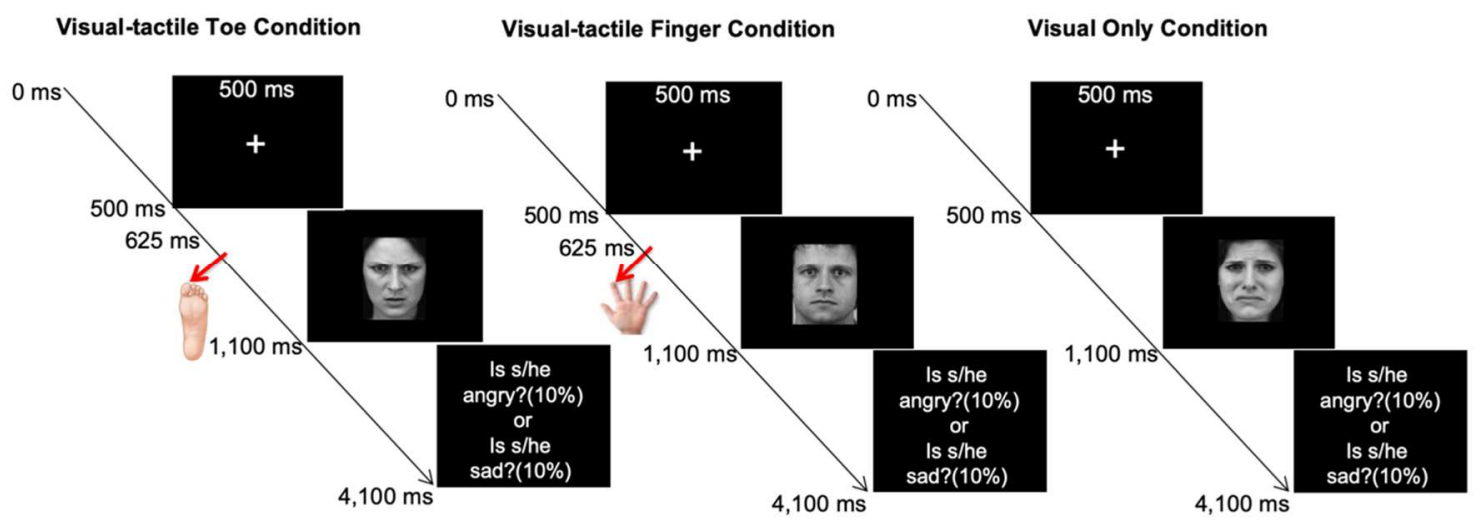

Figure 2

VEP-free SEPS

Finger

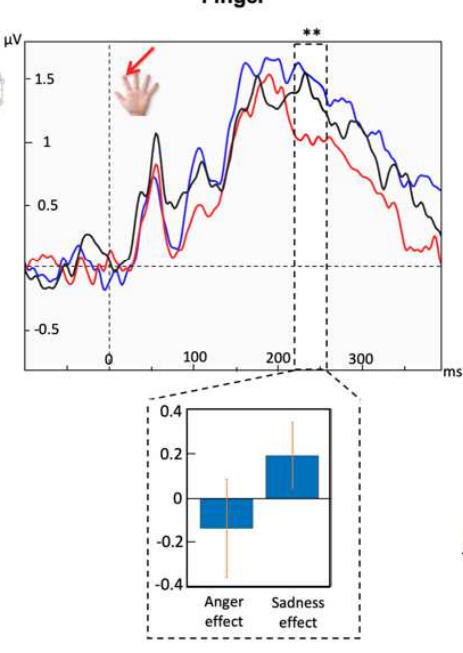

Toe
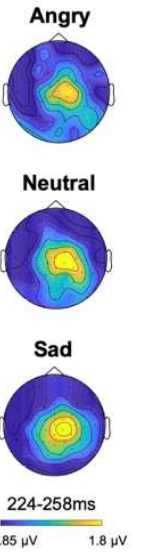
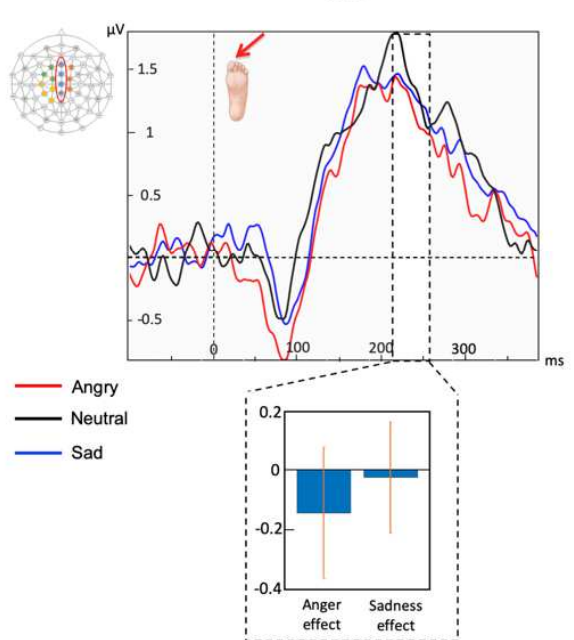

Angry

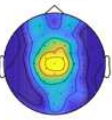

Neutral

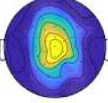

Sad

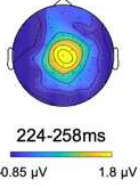


Figure 3

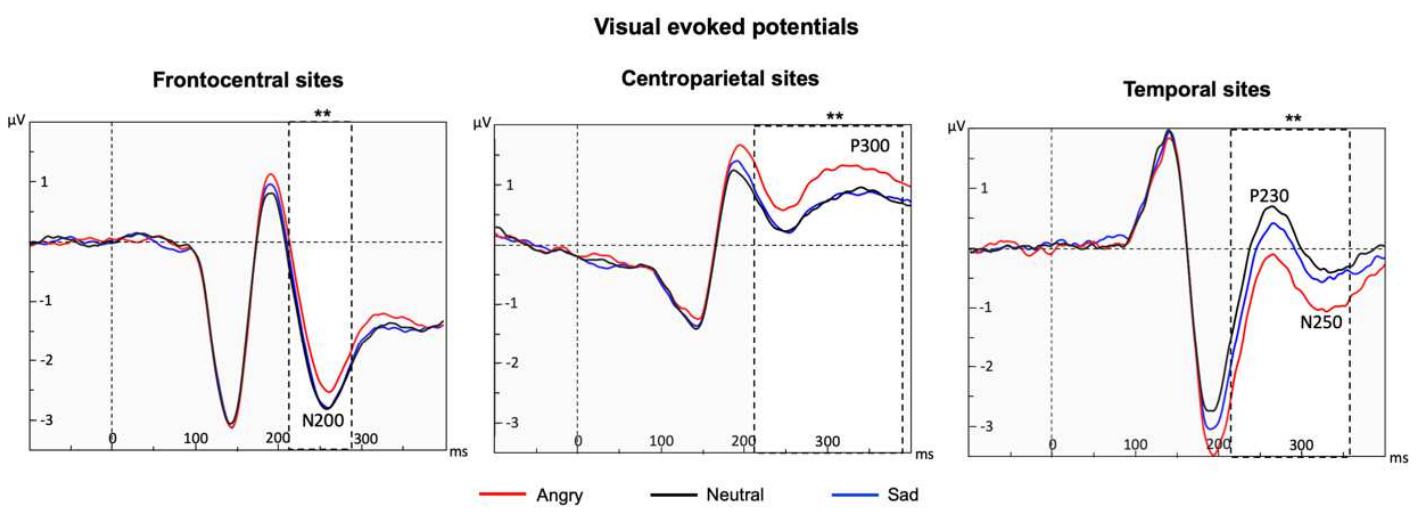

Supplementary Figure

a
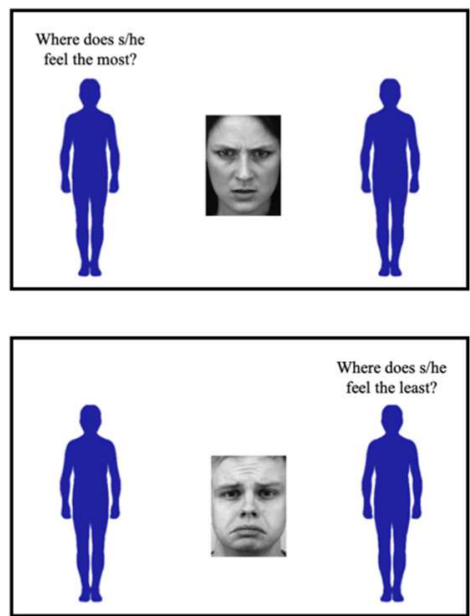

b
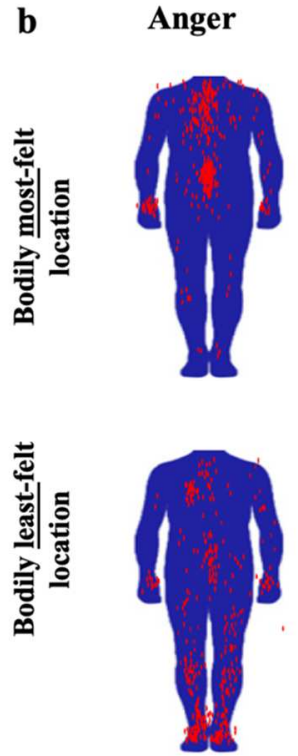

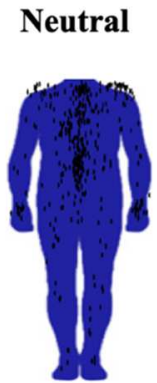

Sadness
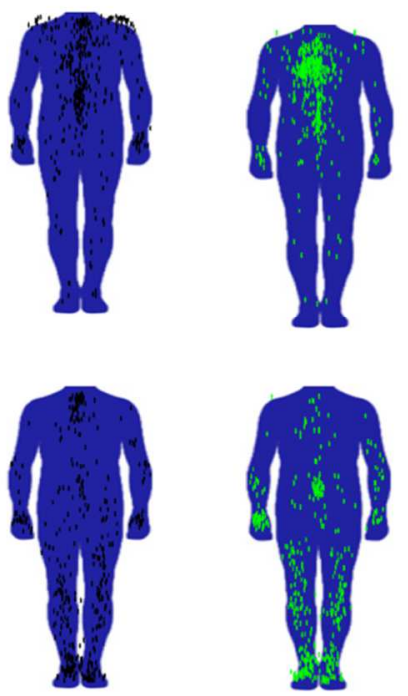

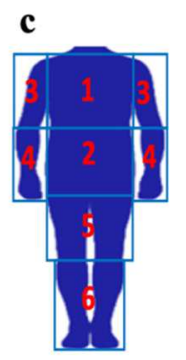

